\title{
ASOCIACIÓN MICORRÍZICA ARBUSCULAR EN PASTIZALES DE LA COMUNIDAD ALTO ANDINA DE CCARHUACCPAMPA - AYACUCHO
}

\section{MYCORRHIZAL ARBUSCULAR ASSOCIATION IN PASTURES OF THE HIGH ANDEAN COMMUNITY OF CCARHUACCPAMPA - AYACUCHO}

\author{
N. Santillana ${ }^{1}$ y M. Toro ${ }^{2}$
}

\begin{abstract}
Resumen
La investigación se realizó con el objetivo de determinar el estado de micorrización de cuatro especies de pastos naturales de la comunidad de Ccarhuaccpampa. Se determinó el número de esporas por $100 \mathrm{~g}$ de suelo, la caracterización de esporas y el porcentaje de colonización de las raíces. El número de esporas/100 g de suelo rizosférico varió de acuerdo a la especie de planta (66 - 732). Destacaron 16 morfotipos de hongos micorrízicos ubicados en tres géneros: Glomus, Acaulospora y Entrophospora, el primero se consideró el más importante por poseer el mayor número de morfotipos y estar presente en los diferentes suelos evaluados. Se comprobó una baja micorrización de las raíces analizadas $(20$ y $27 \%)$ y la presencia de la asociación micorrízica de tipo arbuscular en todas las especies estudiadas. Se determinó alta correlación entre el número de esporas por $100 \mathrm{~g}$ de suelo y el porcentaje de micorrización de las raíces, lo cual indicó similar tendencia entre ambas determinaciones.
\end{abstract}

Palabras clave: hongos arbusculares, colonización, esporas, morfotipos

\begin{abstract}
The aim of this study was to determine the mycorrhizal status of four species of natural pastures of the community of Ccarhuaccpampa. The number of spores per $100 \mathrm{~g}$ of soil, the characterization of spores and the percentage of colonization of the roots were determined. The number of spores / $100 \mathrm{~g}$ of rhizospheric soil varied according to the plant species (66-732). Sixteen morphotypes of mycorrhizal fungi were located in three genera: Glomus, Acaulospora and Entrophospora, the first one was considered the most important because it had the highest number of morphotypes and was present in the different soils evaluated. A low mycorrhization of the analyzed roots was found (20 and $27 \%$ ) and the presence of the arbuscular mycorrhizal association in all the species studied. A high correlation was determined between the number of spores per $100 \mathrm{~g}$ of soil and the percentage of mycorrhization of the roots, which indicated a similar tendency between both determinations.
\end{abstract}

Key words: arbuscular fungi, colonization, spores, morphotypes.

\section{Introducción.}

Las praderas alto andinas de la región de Ayacucho presentan problemas de erosión de suelos debido a la pérdida de cobertura vegetal por sobrepastoreo, ocurriendo la desaparición de especies forrajeras importantes de uso ganadero tales como Festuca, Muhlembergia y Calamagrostis (Durand, 2008). En la actualidad, se tiene conocimiento limitado acerca de los microorganismos que participan en la nutrición de los pastizales naturales, es así que la presencia de la asociación micorrízica de tipo arbuscular aún no ha sido estudiada. La asociación micorrízica se destaca como una de las simbiosis más generalizadas e importantes en los ecosistemas terrestres; esta simbiosis mutualista ejerce gran influencia en la nutrición y tolerancia de las plantas al estrés biótico y abiótico, debido a que se forma un vínculo entre la planta y el suelo permitiendo la absorción de nutrientes de poca movilidad como el fósforo (Barea et al., 2011; Voríšková et al., 2016). Asimismo, disminuyen los efectos negativos de la compactación del suelo, promoviendo la formación y la estabilidad de agregados, mejorando el ambiente, la capacidad invasora y la formación de comunidades vegetales en áreas degradadas (Cavalcanti et al., 2014). No obstante, el establecimiento de la asociación micorrízica en condiciones naturales está influenciada por diferentes causas, tales como factores climáticos, características físico - químicas del suelo, y el manejo agronómico (Perez \& Peroza, 2013). Lozano et al. (2015) mencionan que los suelos con manejo agroecológico brindan características adecuadas para sustentar una mayor densidad de esporas de micorrizas arbusculares.

El análisis de poblaciones de hongos micorrízicos (HM) nativos, puede conducir al uso eficiente en 
pastizales que crecen en condiciones de puna. De esta manera se hace necesaria la realización de estudios básicos que permitan determinar el estado de la micorrización en condiciones naturales que a futuro favorezcan el establecimiento y la conservación de los pastizales.

El experimento se realizó con el objetivo de determinar el estado de micorrización de cuatro especies de pastos naturales de la comunidad de Ccarhuaccpampa.

\section{Materiales y métodos. Ubicación.}

El suelo rizosférico y las raíces de cada una de las especies de pastos naturales en estudio (Festuca rigescens, Muhlenbergia ligularis, Calamagrostis vicunarum y Carex ecuadorica) fueron colectadas de la comunidad de Ccarhuaccpampa del Departamento de Ayacucho ubicada a $4116 \mathrm{msnm}$, entre los $13^{\circ} 25^{\prime}$ 3.78" de latitud sur y los $74^{\circ} 54^{\prime} 21.06^{\prime \prime}$ de longitud oeste. La precipitación total anual fue de $1100 \mathrm{~mm}$, la temperatura máxima y mínima promedio anual fue de 14 y $-2^{\circ} \mathrm{C}$ respectivamente. La precipitación presenta una distribución diferencial a lo largo del año. La máxima precipitación se da durante la época de lluvias que abarca de diciembre a marzo y la época de sequía, de abril a noviembre. La recolección de las muestras se realizó en época de sequía (octubre), a partir de media hectárea de pastizales, considerando tres plantas por especie y extrayendo la totalidad de la zona de raíces más suelo (suelo rizosférico), a una profundidad de 20 $\mathrm{cm}$. Las muestras fueron conservadas en refrigeración hasta su evaluación. El suelo para el análisis de las características físico-químicas fue extraído del área de recolección de las muestras vegetales y presentó las siguientes características: textura Franco-ArcillosoArenoso, pH ácido (5.8), nivel alto de materia orgánica $(6.34 \%)$ y nitrógeno total $(0.31 \%)$ y niveles bajos de fósforo disponible (5.7 ppm) y potasio (98.3 ppm).

La extracción de las esporas, la descripción de los morfotipos y la tinción de raíces se realizó en el laboratorio de Estudios Ambientales del Instituto de Zoología y Ecología Tropical de la Universidad Central de Venezuela.

Tratamientos y diseño estadístico.

Se consideró cuatro tratamientos constituidos por suelos rizosféricos y raíces provenientes de cuatro especies de pastos naturales (Festuca rigescens, Muhlenbergia ligularis, Calamagrostis vicunarum y Carex ecuadorica). Se utilizó el diseño Completamente al Azar con tres repeticiones por tratamiento.

Extracción de esporas y descripción de morfotipos.

La extracción de las esporas de los hongos Glomeromycota se realizó a partir de suelos rizosféricos de las especies de pastos evaluados, utilizando el método de Filtración y Decantación descrito por Gerdemann \& Nicolson (1963).
Los morfotipos encontrados se compararon según las descripciones de especies sugeridas por INVAM (International Culture Collection of (Vesicular) Arbuscular Mycorrhizal Fungi) de la Universidad de Virginia, EE.UU. y del Departamento de Patología Vegetal de la Universidad de Agricultura en Szczecin, Polonia (Stürmer et al. 2013).

Tinción de raíces y cuantificación de colonización.

La tinción de las raíces se realizó utilizando el método descrito por Phillips \& Hayman, (1970), que consiste en el clareo con $\mathrm{KOH}$ al $10 \%$, acidificación con $\mathrm{HCl}$ al $10 \%$ y tinción de raíces con una solución colorante de Azul de Tripano al 0.05\%. Posteriormente se elimina el colorante y se dejan las raíces en una solución de lactoglicerol. Una vez teñidas, se procede a cortar las raíces en segmentos y colocarlas en laminillas utilizando lactoglicerol. Se observan las raíces al microscopio óptico a $40 \mathrm{X}$ de aumento y se registra la frecuencia de las estructuras fúngicas micorrízicas (arbúsculos, vesículas o hifas) en las células corticales y segmentos de raíces.

La cuantificación de la colonización en las raíces teñidas se realizó utilizando los métodos de intersección de cuadrantes y el de evaluación de campos colonizados y no colonizados (Giovanetti \& Mosse, 1980).

Análisis estadístico.

Para el procesamiento de los datos se utilizó un Análisis de Varianza (ANVA) de clasificación simple después de verificar que los supuestos cumplían con el ajuste de homogeneidad de varianza y distribución normal; para ello se empleó el paquete estadístico MINITAB v.16. Las medias fueron comparadas por el test de Tukey para un nivel de significación de $p \leq 0,05$. Mediante el análisis de correlación de Pearson se determinó la relación entre el número de esporas y el porcentaje de raíces micorrizadas

\section{Resultados y discusión.}

De acuerdo con los resultados presentados en la Figura 1, el número de esporas varía según el tipo de suelo rizosférico. El suelo extraído de Festuca rigescens presentó el mayor número de esporas/100 g de suelo seco (732), y superó con diferencias significativas al resto de tratamientos, mientras que Muhlenbergia ligularis presentó el menor número de esporas (66). Ambas especies pertenecen a la familia de las Poaceas, pero $F$. rigescens generalmente crece en suelos poco húmedos y en vegetación tipo pajonal, mientras que $M$. ligularis crece en suelos húmedos y en vegetación tipo césped de puna. La especie Calamagrostis vicunarum también pertenece a la familia Poaceae, es una especie abundante en las zonas de bofedales de puna, o bordes de cursos de agua, y Carex ecuadorica pertenece a las Cyperaceas, crece en 


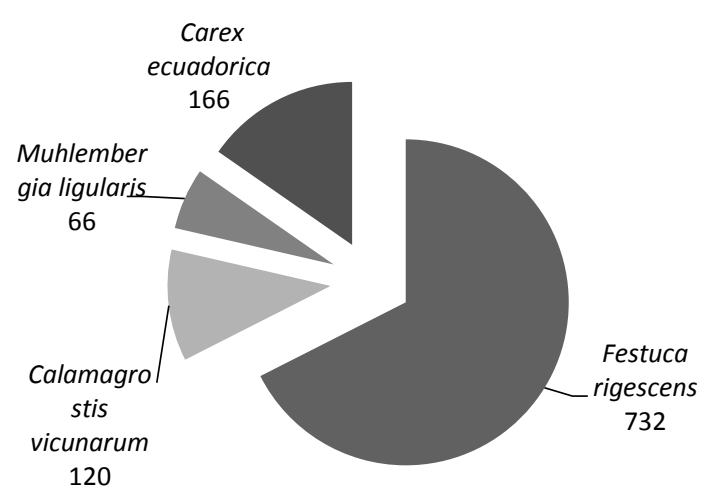

Figura 1. Prueba de Tukey (0.05) del número de esporas de hongos micorrízicos arbusculares en suelo rizosférico de cuatro especies de pastos naturales.

suelos poco húmedos, pajonales y césped de puna (Durand, 2008). Los resultados obtenidos concuerdan con Howeler et al. (1987) quienes registraron conteos de esporas nativas asociadas a Brachiaria decumbens, que no superan las 1000 esporas/100 g de suelo seco. Igualmente, Serralde \& Ramírez (2004) informaron de 400 a 1600 esporas/100 g de suelo seco, con pH ácido, en poblaciones de HMA nativas asociadas al cultivo del maíz. Medina et al. (2010) indican entre 800 y 1700 esporas por gramo de suelo con $\mathrm{pH}$ salino. Perez \& Peroza (2013) determinaron entre 500 a 1700 esporas en pasto angleton (Dichathium aristatum Benth).

Se han determinado 16 morfotipos de esporas pertenecientes a 3 géneros: Glomus, Acaulospora y Entrophospora, (Figura 2) siendo Glomus el que presentó el mayor \% de morfotipos (69\%), seguido de Acaulospora (25\%) y Entrosphopora (6\%) (Figura 3). En las especies Festuca rigescens, Muhlenbergia ligularis y Calamagrostis vicunarum se observaron los géneros Glomus y Aucalospora, mientras que en Carex ecuadorica, los géneros Glomus y Entrophospora. Al respecto, Medina et al. (2010) observaron cuatro de los 19 géneros conocidos (Glomus, Acaulospora, Gigaspora y Scutellospora) e indican que el más común fue Glomus, al cual pertenecieron 21 morfotipos, seguido por Acaulospora, con cinco de los morfotipos encontrados. Asimismo, indican que los géneros menos frecuentes fueron Gigaspora y Scutellospora, en los que solo se identificó un morfotipo en cada uno. No reportaron la presencia de Entrophospora. Marinho (2014) registra 80 taxones de hongos micorrízicos arbusculares, distribuídos en 16 géneros, con predominancia de Glomus y Acaulospora en suelos naturales de Brasil. Los resultados evidencian que el género Glomus predomina y se adapta a las condiciones edafoclimáticas de Ccarhuaccpampa ubicada a $4116 \mathrm{msnm}$ ( $\mathrm{pH}$ ácido, bajas temperaturas, precipitación estacional). Al respecto, Medina et al. (2010) indican a Glomus como género dominante en la mayoría de las poblaciones nativas y es posible que se encuentre en simbiosis activa en mayor proporción que

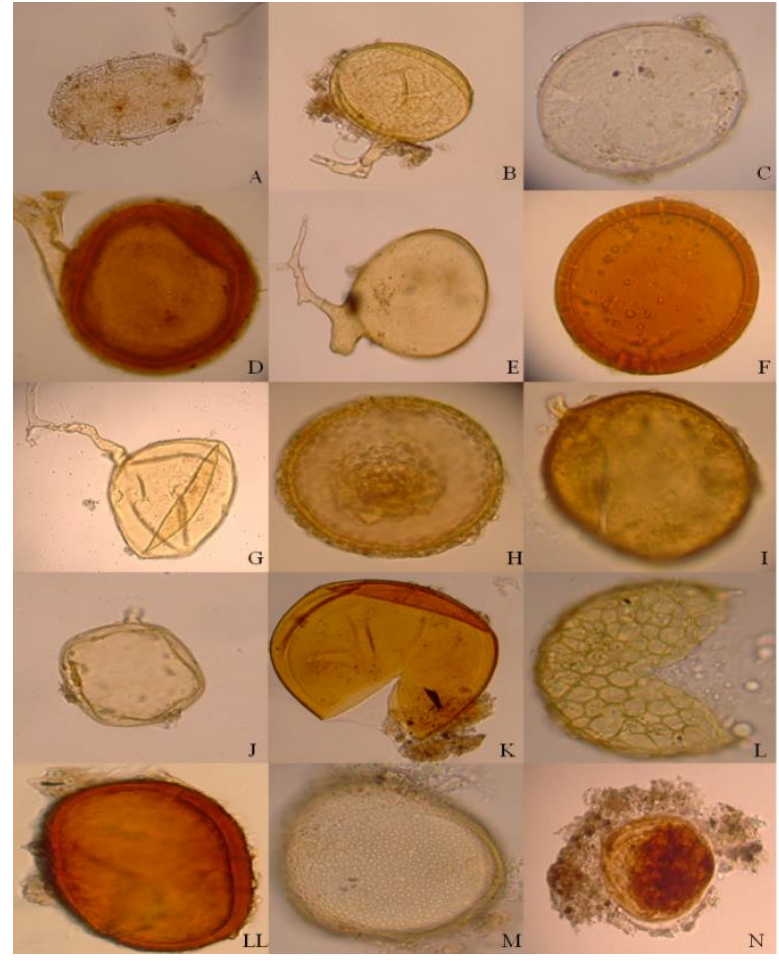

Figura 2. Morfotipos de esporas de hongos micorrízicos arbusculares, género Glomus (A-J), género Acaulospora (K-M), género Entrophospora (N).

los demás, lo que refleja su capacidad de adaptación a diferentes condiciones edáficas y ofrece un alto potencial para su uso como biofertilizante. Figueira et al. (2016) identificaron especies de Glomus y Acaulospora en suelos con $\mathrm{pH}$ entre 4.08 y 5.89, similares a los suelos evaluados. Trinidad-Cruz et al. (2017) reportan también especies de Glomus y Acaulospora como las más frecuentes en suelos de Agave cupreata. Meza et al. (2017) indican el género Glomus como la especie prevalente en plantaciones forestales de melina (Gmelina arborea) en la zona central del trópico húmedo ecuatoriano. Asimismo, en

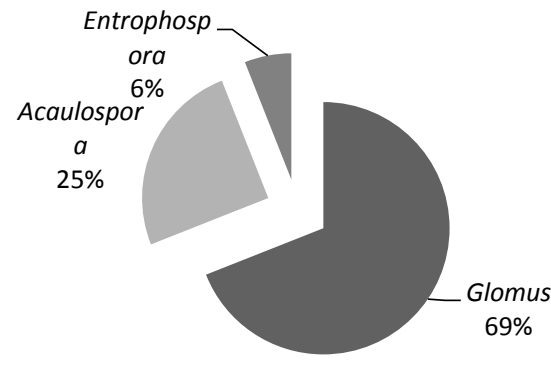

Figura 3. Porcentaje de morfotipos por género de hongo micorrízico arbuscular. 
la rizósfera de frutales mencionan la dominación de esporas del género Glomus (Dantas et al., 2015).

De acuerdo con los resultados obtenidos en el porcentaje de colonización de las raíces (Figura 4), se determinó que la asociación micorrízica presente en las raíces de las cuatro especies evaluadas, es arbuscular, debido a que fueron identificadas tanto las vesículas como los arbúsculos, estructuras características de este tipo de asociación. Los porcentajes de colonización de las raíces variaron entre 20 y $27 \%$, sin diferencias significativas entre ellos, lo que demuestra entre baja (<25) a mediana (>25) micorrización de las raíces analizadas, en comparación con la colonización micorrizica descrita por Mora et al. (2012) para pasturas tropicales (68\%), posiblemente debido a las condiciones edafoclimáticas del lugar de estudio y a la época de muestreo (sequía). Al respecto, Voríšková et al. (2016) indican que el desarrollo de los hongos micorrízicos en las raíces es influenciado por la especie de planta y por las condiciones abióticas del suelo, asimismo, Guadarrama et al. (1998) indican que en época de sequía la productividad de raíces finas decrece, disminuyendo el porcentaje de infección.

En la presente investigación, resalta la alta correlación (0.972) con valor de $\mathrm{P}=0.028$, entre el número de esporas por $100 \mathrm{~g}$ de suelo y el porcentaje de micorrización de las raíces, indicando similar tendencia en la determinación del número de esporas y del porcentaje de infección de las raíces en las especies vegetales evaluadas, posiblemente debido a la influencia de la especie vegetal Festuca rigescens, que de acuerdo a lo observado, presenta mejor capacidad micotrófica en relación a las otras especies (Sene et al. 2012).

Asimismo, es importante indicar la abundancia y dominancia del género Glomus, asociado a las cuatro especies vegetales evaluadas y su capacidad de adaptación a las condiciones de Ccarhuaccpampa, por lo que es necesaria su conservación en estos espacios.

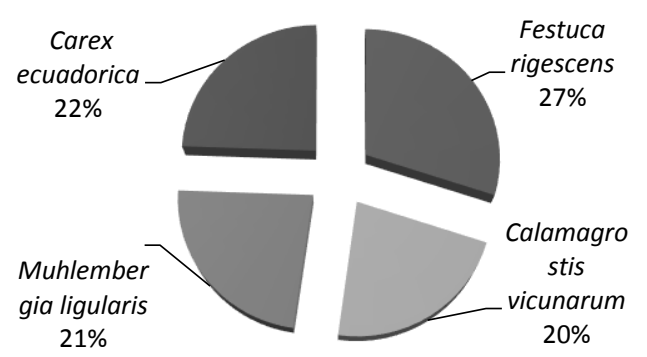

Figura 4. Porcentaje de raíces micorrizadas por especie de planta.

\section{Agradecimientos.}

A la Red BIOFAG (CYTED - España) y al Proyecto FOCAM- UNSCH - Perú por el apoyo económico brindado para la realización de la presente investigación en el Laboratorio de Estudios Ambientales de la Universidad Central de Venezuela.

\section{Literatura citada.}

Barea J.M., Palenzuela J., Cornejo P., Sánchez-Castro I, Navarro-Fernández C., Lopéz-García A., Estrada B., Azcón R., Ferrol N. \& Azcón-Aguilar C. 2011. Ecological and functional roles of mycorrhizas in semiarid ecosystems of Southeast Spain. Journal of Arid Environments. 75:1292-1301.

Cavalcanti A., Vanusa M., Pereira M., Barreto M., Ribeiro M., Costa M. 2014. Caracterização morfológica e molecular de fungos micorrízicos arbusculares isolados de áreas de mineração de gesso, Araripina, PE, Brasil. Hoehnea 41(3): 393-400.

Dantas B. Baller O., Pereira J., Guimarães A., Pagano M. 2015. Diversidade de fungos micorrízicos arbusculares em pomar orgânico no semiárido cearense. Ciência Rural, Santa Maria. 45(8):1480-1486.

Durand F. 2008. Fenología de Diez especies de pastos naturales de los pastizales altoandinos de la comunidad de Ccarhuaccpampa - Ayacucho. Tesis título de Ingeniero Agrónomo. Facultad de Ciencias Agrarias. Universidad Nacional de San Cristóbal de Huamanga, Ayacucho - Perú 112 p.

Figueira C., Pereira M., dos Santos V., Miguel D., Ribeiro E. 2016. Fungos micorrízicos arbusculares: composição, comprimento de micelio extrarradicular e glomalina em áreas de mata Atlântica, Rio de Janeiro. Ciência Florestal, Santa Maria. 26(2): 419 - 433 .

Gerdemann J. W. \& Nicolson T. H. 1963. Spores of mycorrhizal endogone species extracted from soil by wet sieving and decanting. Transaction of the British Mycological Society. 46: 235-244.

Giovannetti M. \& Mosse B. 1980. An evaluation of techniques for measuring vesicular arbuscular mycorrhizal infection in roots. New Phytologist. 84: 489500 .

Guadarrama C.P., Sánchez-Gallén I. \& Álvarez-Sánchez. P.J. 1998. Ecología de hongos micorrízicos arbusculares en la selva y pastizales de los Tuxtlas en Veracruz. p.27-39. En: Avances de la Investigación micorrízica en México. Zulueta R.R., Escalona A.M., Trejo A.D.(eds). Universidad Veracruzana, 1ra. Edición, México. 283 p.

Howeler R. H., Sieverding E. \& Saif S. 1987. Practical aspects of mycorrhizal technology in some tropical crops and pastures. Plant and Soil. 100:249-283.

Lozano S. J., Armbrecht I., Montoya L.J. 2015. Hongos formadores de micorrizas arbusculares y su efecto sobre la estructura de los suelos en fincas con manejos agroecológicos e intensivos. Acta Agron. 64(4): 289-296.

Marinho F. 2014. Padrão de distribuição de fungos micorrízicos arbusculares e atividade microbiana do solo no Parque Nacional do Catimbau-PE/ Frederico Marinho-Recife. Tesis de Maestria. Universidad Federal de Pernambuco. Centro de Ciências Biológicas - Brasil $77 \mathrm{p}$. 
Medina L., Rodriguez Y., Torres Y. \& Herrera R. 2010. Aislamiento e identificación de hongos micorrícicos arbusculares nativos de la zona de Las Caobas, Holguín. Cultivos Tropicales (La Habana). 31:33-42.

Meza B.F., Díaz O.E., Escobar T.H., Belezaca C., Cachipuendo C.J., Meza B.G., López M.F., Meza B. C., Meza B.J., Cachipuendo C.J., Cabrera V.R. 2017. Identificación de Hongos Micorrízicos en Plantaciones de Melina (Gmelina arborea Roxb) en el Trópico Húmedo Ecuatoriano. Rev Inv Vet Perú. 28(4): 969-975.

Mora E., Toro M. \& López-Hernández D. 2012. A Survey of arbuscular mycorrhizae, Rhizobium and phosphate solubilizing bacteria in low fertility savanna soils in Central Venezuela (Estación Experimental La Iguana). En: M. Miransari (ed), Soil Microbiology. Nova Science Publishers Inc.

Pérez C. A. \& Peroza C. V. 2013. Micorrizas arbusculares asociadas al pasto angleton (Dichathium aristatum Benth) en fincas ganaderas del municipio de Tolú, SucreColombia. Revista MVZ (Córdova). 18: 3362-3369.

Phillips J.M. \& Hayman D.S. 1970. Improved procedures for clear- ing roots and staining parasitic and vesiculararbuscular mycor- rhizal fungi for rapid assessment of infection. Transactions of the British Mycological Society. 55: 158-161.
Sene G., Samba-Mbaye R., Thiao M., Khasa D., Kane A. 2012. The abundance and diversity of legume-nodulating rhizobia and arbuscular mycorrhizal fungal communities in soil samples from deforest and man-made forest systems in a semiarid Sahel region in Senegal. European Journal of Soil Biology 52: 30 - 40

Serralde A. M. \& Ramírez M. M. 2004. Análisis de poblaciones de micorrizas en maíz (Zea mays) cultivado en suelos ácidos bajo diferentes tratamientos agronómicos. Revista Corpoica. 5: 31-40.

Stürmer S.L., Stürmer R. \& Pasqualini D. 2013. Taxonomic diversity and community structure of arbuscular mycorrhizal fungi (Phylum Glomeromycota) in three maritime sand dunes in Santa Catarina state, south Brazil. Fungal Ecology. $6: 27-36$.

Trinidad-Cruz J., Quiñones-Aguilar E., Hernández-Cuevas L., López-Pérez L., Rincón-Enríquez G. 2017. Hongos micorrízicos arbusculares asociados a la rizosfera de Agave cupreata en regiones mezcaleras del estado de Michoacán, México. Scientia Fungorum, 45: 13-25

Voríškováa A., Janouškováa M., Slavíkovác R., Pánkováa H., Ondrej D., Vazacováa K., Rydlováa J., Vosátkaa M. \& Münzbergováa Z. 2016. Effect of past agricultural use on the infectivity and composition of a community of arbuscular mycorrhizal fungi Agriculture. Ecosystems and Environment. 221:28-39.

${ }^{1}$ Laboratorio de Rhizobiología, Facultad de Ciencias Agrarias, Universidad Nacional de San Cristóbal de Huamanga. Ayacucho, Perú. nerysantillana@yahoo.es.

${ }^{2}$ Laboratorio de Estudios Ambientales del Instituto de Zoología y Ecología Tropical de la Facultad de Ciencias de la Universidad Central de Venezuela. 